\title{
Case Study on Retrofit of Steel Plate Shear Walls Using Low Yield Point Steel Infill Plates
}

Amir Reza Khoeilar, Tadeh Zirakian*, David Boyajian, Sami Maalouf, and Nazaret Dermendjian

Department of Civil Engineering and Construction Management, California State University, Northridge, CA, USA

\begin{abstract}
Steel plate shear walls (SPSWs) have been frequently used in seismic design and retrofit of buildings over the past three decades or so. Employment of infill plates made of low yield point (LYP) steel with considerably low yield stress and high elongation capacity is believed to provide the possibility in order to improve the structural and seismic characteristics of such lateral force-resisting systems. Among the various benefits is the early yielding of LYP steel infill plates, which can result in greater energy absorption capacity and limitation of the plastic deformation demand to the surrounding frame structure. On this basis, a case study is performed using numerical simulations and reported in this paper on the seismic retrofit of SPSWs using LYP steel infill plates of double thickness. It is shown that the retrofit of a steel shear wall using a LYP steel infill plate of double thickness can result in desirable plate-frame yielding sequence and interaction. Moreover, this retrofit strategy can improve the initial stiffness, buckling stability, and energy dissipation capacity of the existing SPSW system.
\end{abstract}

Keywords: Steel plate shear wall; Retrofit; Low yield point steel; Structural behavior; Seismic performance; Numerical simulation

\section{Introduction}

Steel plate shear walls (SPSWs) find frequent use in the United States, Japan, and Canada, resulting in a considerable amount of theoretical and experimental research activity on these systems. Attention has been focused to the structural behavior of SPSWs with concerted efforts on analytical models as lateral force-resisting systems in the design of low-, medium-, and high-rise buildings against seismic and wind loads. The advantages of using SPSWs in such a manner in buildings include stable hysteretic characteristics, high plastic energy absorption capacity, and enhanced stiffness, strength, and ductility [1].

SPSWs have been used with two different design philosophies as well as detailing strategies. One approach employs heavily-stiffened SPSWs to ensure that the wall panel achieves its full plastic strength prior to failing as a result of out-of-plane buckling. Thus, the stiffened wall panels have been found to resist large lateral forces and are capable of dissipating harmful earthquake-induced energy effects. Such systems are currently used in practice in Japan, where high-fabrication costs are tolerated in exchange for heightened seismic and structural performances of their buildings. The North American practice, on the other hand, uses thin unstiffened steel wall plates, which has been shown to exhibit nonlinear behavior during out-of-plane buckling at relatively small story [2]. The elastic shear buckling of the thin plate in SPSWs usually results in reduced stiffness, strength, and energy dissipation capacities. Although the tension field action is capable of providing the post-buckling strength, the occurrence of shear buckling induced during the early stages of excitation in even small or moderate earthquakes, has been known to result in permanent, out-of-plane, deformations that, in turn, adversely affects the serviceability of such elements [3]. While infill plates may be implemented in either stiffened or unstiffened forms, depending on the design philosophy chosen, the latter technique has found more common usage in North American practices overall [4].

Buckling stability, energy dissipation capacity, and serviceability of SPSW systems can be improved by either increasing the web thickness or using horizontal and vertical stiffeners. Nevertheless, this may not result in an economical design of shear walls with conventional steel infill plates. The advantages of application of low yield point (LYP) steel in SPSW systems have been demonstrated through several studies [5]. Conducted nonlinear inelastic analyses and showed that low yield steel shear walls can behave better than the standard constructional grade steel shear walls under extreme seismic conditions. Based on nonlinear dynamic analyses, De Matteis et al. [6] demonstrated that low-yield shear panels may strongly enhance the seismic performance of steel frames and can also supply a large source of energy dissipation, which results in a limitation of plastic deformation demand to the primary structure. Through experimental programs, Vian and Bruneau [7] and Tsai and Lin [8] showed that the lower yield strength of a LYP steel shear wall can result in earlier onset of energy dissipation by the panel as compared to a hot-rolled plate. The lateral force-resisting and energy dissipating capabilities of LYP steel shear walls were studied and verified through a numerical study reported by Lashgari [9]. Moreover, the beneficial behavior of low yield steel panels with respect to ordinary steel panels as well as the improved seismic behavior of existing structures retrofitted by shear wall panels were verified through a numerical study performed by Mistakidis [10]. In other experimental investigations by Chen and Jhang $[6,11]$, it was demonstrated that the LYP steel shear wall system has excellent deformation and energy dissipation capacities. Most recently, the various advantages of use of LYP steel plates in seismic design and retrofit of conventional steel shear wall systems were demonstrated in a series of numerical studies reported by Zirakian and Zhang [12,13] and Zhang and Zirakian [14].

It is noted that low yielding plates can also be employed in momentresisting and braced frames as energy-dissipative components.

*Corresponding author: Tadeh Zirakian, Department of Civil Engineering and Construction Management, California State University, Northridge, CA, USA Tel:+1-818-677-7718; Fax:+1-818-677-5810; E-mail: tadeh.zirakian@csun.edu

Received November 28, 2015; Accepted December 18, 2015; Published December 28, 2015

Citation: Khoeilar AR, Zirakian T, Boyajian D, Maalouf S, Dermendjian N (2015) Case Study on Retrofit of Steel Plate Shear Walls Using Low Yield Point Steel Infill Plates. J Steel Struct Constr 2: 106. doi:10.4172/2472-0437.1000106

Copyright: (C) 2015 Khoeilar AR, et al. This is an open-access article distributed under the terms of the Creative Commons Attribution License, which permits unrestricted use, distribution, and reproduction in any medium, provided the original author and source are credited. 
al. [15] reported a relevant study on the application of steel bracings for retrofitting of a reinforced concrete frame and discussed the advantages and disadvantages of adopting three alternative bracing configurations. Nevertheless, further studies need to be performed on the influence of the space distribution, or in other words, distribution patterns of such energy-dissipative components throughout the structures.

Due to the low yield stress nature of the LYP steel, material yielding in LYP steel shear walls may occur prior to the occurrence of their buckling geometrically. Hence, an accurate evaluation of buckling and yielding interactions of SPSWs can result in a more efficient structural and economical design of these systems. Accordingly, infill plates in SPSWs may be qualitatively and quantitatively classified as slender, moderate, and stocky based on their slenderness parameter as well as geometrical-material bifurcation characteristics [16]. The following is a summary of plate failures based on such criteria: slender plates undergo early elastic buckling and subsequently yield in the post-buckling stage; moderate plates undergo simultaneous buckling and yielding; stocky plates yield first and then undergo post-yield inelastic buckling.

In this study, the structural performance of code-designed and unstiffened LYP steel shear wall systems is examined primarily through finite element analysis. The advantages of using LYP steel in SPSW systems as compared to conventional steels are demonstrated through comparative studies herein.

\section{Design of Steel Shear Wall Models}

In order to design shear wall models, we consider six single-story, single-bay, and full-scale steel shear walls with $2000 \times 3000,3000 \times 3000$, and $4500 \times 3000 \mathrm{~mm}$ infill plates of various slenderness ratios and steels as designed in accordance with the seismic provisions of AISC 341-10 [17]. Specifications of the code-designed SPSW models are provided in Table 1 , in which $l, h$ and $t_{p}$ are the length, height, and thickness of the infill plate, respectively.

SPSW specifications are prescribed according to capacity-design principles whereby boundary elements permit web plates to develop significant diagonal tension. In fact, horizontal and vertical boundary elements, i.e., HBEs (beams) and VBEs (columns), are designed to elastically resist developing the full yield strength of the infill plate. By such a measure, the infill plate yields in tension before the boundary elements are able to develop plastic hinges [18]. As can be seen in Table 1, ASTM A572 Gr. 50 steel with $345 \mathrm{MPa}$ yield stress is selected for the boundary frame, and LYP 100 and ASTM A36 steel with respective 100 and $250 \mathrm{MPa}$ yield stresses are selected for the infill plates. In order to design the boundary frame members in SPSWs, corresponding infill plate thicknesses are initially determined. The following equation is derived by setting the critical shear stress $\left(\tau_{c r}\right)$ of a rectangular clamped plate to that of the plate shear yield stress $\left(\tau_{y p}=\sigma_{y p} / \sqrt{3}\right)$ in accordance to the von Mises yield criterion. The accuracy of the theoretical predictions corresponding to a concurrent geometrical-material bifurcation condition is verified by numerical results, as presented in subsequent sections. Following the determination of the infill plate thickness of the SPSW4 model, infill plate thicknesses of the SPSW2/3 and SPSW 5 models were chosen to represent slender-web and stockyweb steel shear wall system behaviors, respectively.

$$
t_{\mathrm{p}-\text { limit }}\left(=t_{p-\text { SPSW }}\right)=b \times \sqrt{\frac{12 \times\left(1-v^{2}\right) \times \sigma_{y p}}{\left(8.98+5.6 /(a / b)^{2}\right) \times \pi^{2} \times E \times \sqrt{3}}}
$$

Also, as shown in Table 1, the SPSW2 model for both LYP100 and ASTM A36 steel material results in identical sections for the boundary frame members. This is due to the HBE and VBE designs being dominated by a specified stiffness, rather than a strength criterion, that is independent of material type.

\section{Details of Finite Element Modeling}

ANSYS 11.0 [19] was utilized to develop and analyze the SPSW numerical models under monotonic and cyclic loadings. Boundary frame members as well as infill plates of the steel shear walls were modeled by the Shell181 element. This four-node element with six degrees of freedom at each node is suitable for analyzing thin to moderately-thick shell structures and for treating linear, large rotations and/or large strain nonlinear applications. The SPSW1, SPSW4, and SPSW6 finite element models are illustrated in Figure 1.

As shown in Figure 1, both columns are fully fixed at their bases and the exterior nodes of the column flange and stiffener elements around the perimeter of the panel zones are restrained against out-ofplane displacement. Moreover, as seen in Figure 1c, HBEs in SPSW6 model with the largest span length are braced at their mid-span against lateral displacement. Details of the respective steel material selected for the boundary frame members and infill plates of the SPSW models are given in Table 1.

Figure 2 displays the stress-strain relationships as well as the mechanical properties of the various steel materials that were applied in formulating the finite element models. Furthermore, the von Mises yield criterion was used for material yielding, and isotropic and kinematic hardening rules were incorporated in the respective nonlinear pushover and cyclic analyses. In order to account for initial imperfections, very small out-of-plane deformations of about $\sqrt{l \times h} / 1000$ and proportional to the lowest eigen-mode shape of elastic buckling were introduced to the SPSW models.

Validation of the SPSW numerical models was accomplished by considering the experimental results of two specimens as tested by researchers Lubell [20] and Chen and Jhang [11], whose studies involved SPSWs with respective slender conventional steel and stocky LYP steel infill plates. The comparison details with the aforementioned pair of experimental results are illustrated in Figures $3 a-3 b$ respectively. As can be seen, there is close agreement between the numerical and

\begin{tabular}{|c|c|c|c|c|c|c|}
\hline \multirow[t]{2}{*}{ Model } & \multicolumn{2}{|c|}{ Infill Plate } & \multirow[t]{2}{*}{ HBE (Beam) } & \multirow[t]{2}{*}{ VBE (Column) } & \multicolumn{2}{|c|}{ Design Steel Type } \\
\hline & $l \times h \times t_{p}(\mathbf{m m m m} \times \mathbf{m m})$ & Type & & & Frame & Plate \\
\hline SPSW1 & $2000 \times 3000 \times 10.6$ & Moderate & $W 14 \times 120$ & $W 14 \times 311$ & ASTM A572 Gr. 50 & LYP100 \\
\hline SPSW2 & $3000 \times 3000 \times 4.7$ & Slender & $W 14 \times 120$ & W14×132 & ASTM A572 Gr. 50 & ASTM A36, LYP100 \\
\hline SPSW3 & $3000 \times 3000 \times 9.3$ & Slender & W14×233 & $W 14 \times 257$ & ASTM A572 Gr. 50 & LYP100 \\
\hline SPSW4 & $3000 \times 3000 \times 14.0$ & Moderate & $W 14 \times 311$ & $W 14 \times 342$ & ASTM A572 Gr. 50 & LYP100 \\
\hline SPSW5 & $3000 \times 3000 \times 18.7$ & Stocky & W14×398 & W14×426 & ASTM A572 Gr. 50 & LYP100 \\
\hline SPSW6 & $4500 \times 3000 \times 15.8$ & Moderate & $W 30 \times 391$ & $W 14 \times 370$ & ASTM A572 Gr. 50 & LYP100 \\
\hline
\end{tabular}

Table 1: Specifications of code-designed SPSW models (with moderate infill plates highlighted). 

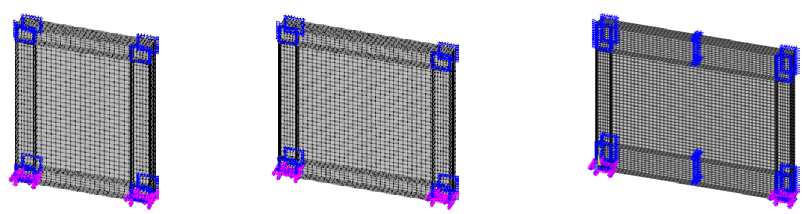

Figure 1: Finite element models (SPSW1, SPSW4, and SPSW6).

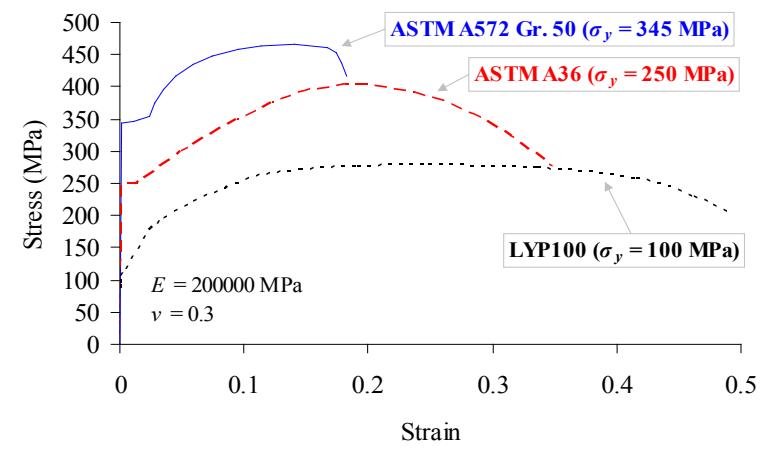

Figure 2: Material properties of the steel used in SPSWs.

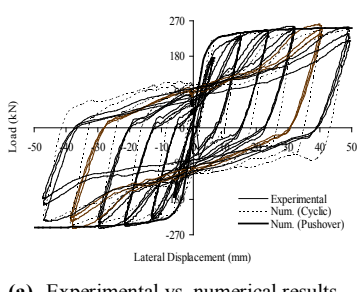

(a) Experimental vs. numerical results (Lubell, 1997)

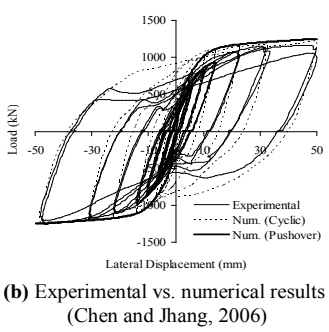

(Chen and Jhang, 2006)
Figure 3: Validation of finite element modeling.

experimental results in both cases.

\section{Discussion of Results}

As the name suggests, LYP steel has a low yield stress of about 90$120 \mathrm{MPa}$, approximately a third the value of that of conventional ASTM A36 steel. This special low yield feature ensures an earlier yielding of the structure and, consequently, reduces the forces being imposed on the frame members to achieve a more enhanced lateral force-resisting and energy dissipating system for use in buildings. In fact, it has been noted that using LYP steel infill plates as recommended herein with double the thickness is not only easier to design, but is a safeguard against the frame from collapsing before the infill plate reaches its ultimate strength [11]. LYP steel shear walls may also be utilized to retrofit existing frame buildings requiring additional strength and stiffness.

\section{Effects of various web-plate materials and thicknesses}

In order to achieve the objectives of this study, the infill plate thickness in the SPSW2 model was increased from $4.7 \mathrm{~mm}$ to 18.7 $\mathrm{mm}$ and the structural behavior along with its components were investigated through finite element analyses. It is noted that the boundary frame members in SPSW2 model were originally designed for $4.7 \mathrm{~mm}$ LYP and conventional steel slender web-plates as shown in Table 1. The lateral load versus out-of-plane displacement and drift ratio curves of the SPSW2 model with respective 4.7, 9.3, 14.0, and $18.7 \mathrm{~mm}$ infill plates are shown in Figures $4 \mathrm{a}-4 \mathrm{~d}$. The out-of-plane displacement-lateral load curves exhibit the buckling behavior of the members, which demonstrate the in-plane stiffness and strength performances of the SPSW system. The points of first yield for the plate and frame are denoted by P.Y. and F.Y., respectively, and E.B. stands for elastic buckling.

As shown in Table 1, the limiting plate thickness corresponding to simultaneous buckling and yielding of a $3000 \times 3000$ [ $\mathrm{mm} \times \mathrm{mm}]$ LYP steel infill plate is estimated to be $14.0 \mathrm{~mm}$, so consideration of thicknesses below and above this limit can result in different buckling and yielding behaviors, i.e., in smaller thicknesses buckling will occur before yielding and in larger thicknesses yielding will take place prior to buckling. On this basis and as seen in Figure 4, infill plates in the SPSW2-4.7 and SPSW2-9.3 models yield in the post-buckling stage. The infill plate in the SPSW2-14.0 model, on the other hand, undergoes simultaneous buckling and yielding as expected, while that of the SPSW2-18.7 model yields prior to buckling.

It is clearly observed that increasing the infill plate thickness reduces the interval between the plate and frame first yield points. However, yielding of the LYP steel infill plates in all cases occurs in advance of the frame yielding due to the low yield stress nature of the material used, while yielding of the conventional steel infill plates in the SPSW214.0 and SPSW2-18.7 models (Figures $4 \mathrm{c}$ and $4 \mathrm{~d}$ ) occurs unfavorably after the onset of frame yielding. As seen in Figures $4 \mathrm{c}$ and $4 \mathrm{~d}$, early yielding of the frame members, especially for the columns, results in significant reductions in both the stiffness and strength characteristics that render a subpar performance of the system as a whole. As already mentioned, since the LYP steel shear walls undergo early yielding, they consequently exhibit larger inelastic deformations as compared to the more conventional steel shear walls having identical infill plate thicknesses. This can be of great importance in seismic design of SPSW systems since the earthquake input energy can be absorbed through plastic deformations of the LYP steel infill plates, which, in turn, relieves the degree of plastic deformation that would otherwise be imparted to the frame of the structure.

\section{Strength and stiffness performances of SPSW models}

The behavior and performance of the SPSW-4.7 and SPSW-9.3 models is next considered. Recall that the SPSW2-4.7 model of ASTM

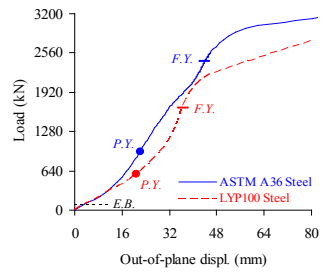

(a) SPSW2-4.7

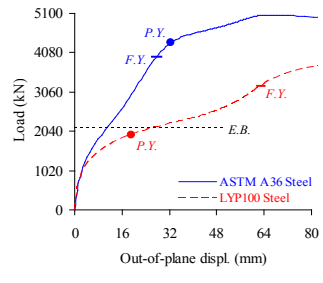

(c) SPSW2-14.0

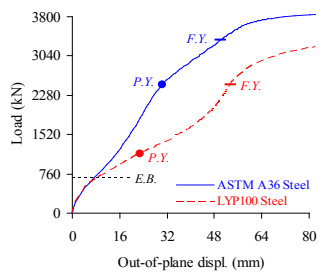

(b) SPSW2-9.3

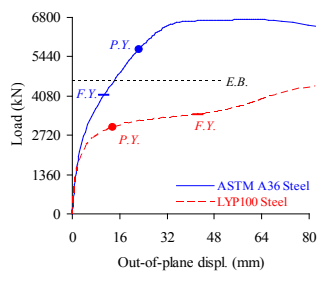

(d) SPSW2-18.7
Figure 4: Structural behavior of SPSW2 model with various infill plate thicknesses and steel types. 
A36 steel was considered as a typical slender-web infill system. Such a member would need to be replaced after an earthquake event if the member had sustained damage. Possible replacements could be achieved by either a $9.3 \mathrm{~mm}$ ASTM A36 member or a LYP100 steel plate. The structural behavior as well as stiffness performance of the SPSW system with the original and two alternative infill plates are shown in Figures 5 and 6, respectively.

From Figures 5 and 6, it is quite evident that the overall performance of SPSW2-4.7-ASTM A36 and SPSW2-9.3-LYP100 models is pretty similar. Note that in Figure 6, due to a larger plate thickness, the SPSW2-9.3-LYP100 model possesses a higher initial stiffness than its SPSW2-4.7-ASTM A36 counterpart which is truly shortlived due to its early yielding nature. As a result, the stiffness of both models is seen to behave quite similarly in spite of their differences with respect to initial stiffness.

\section{Plate-frame interaction}

The von Mises stress contour plots of the SPSW2-4.7-ASTM A36, SPSW2-9.3-LYP100, and SPSW2-9.3-ASTM A36 models at 0.01 and 0.02 drift ratios are shown in Figure 7 with yielded zones in the boundary frame members displayed in red. Note the greater incidence of yielded points in the boundary frame members at a 0.02 drift ratio as compared to a 0.01 value due to increased deformation and force effects. Also, due to the effect of the diagonal tension field action, yielding zones are confined to the HBE and VBE ends in the vicinity of connections where plastic hinges are expected to form. Note that the stress contours and yielding patterns in the boundary frame members of the SPSW2-4.7ASTM A36 and SPSW2-9.3-LYP100 models exhibit similar behavior at both levels of drift ratio, while the HBEs and VBEs in the SPSW2-9.3ASTM A36 model are contrastingly different. It is significant to note

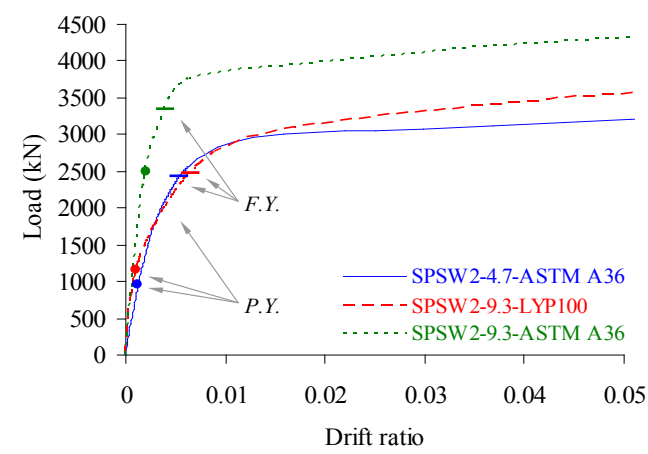

Figure 5: Structural strength and behavior.

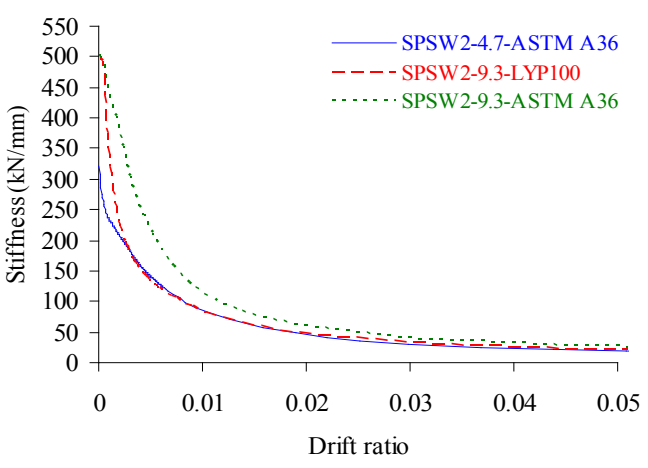

Figure 6. Stiffness performance.

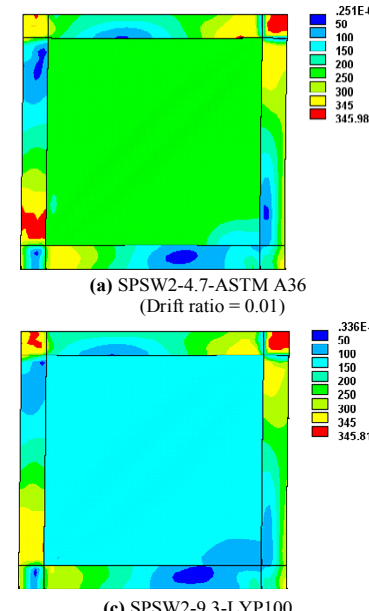

(c) SPSW2-9.3-LYP100

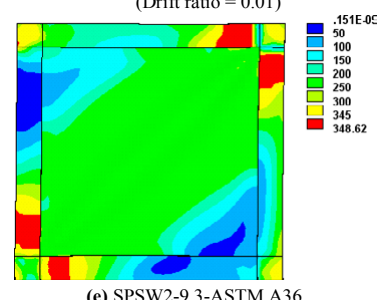

(e) SPSW2-9.3-ASTM A36

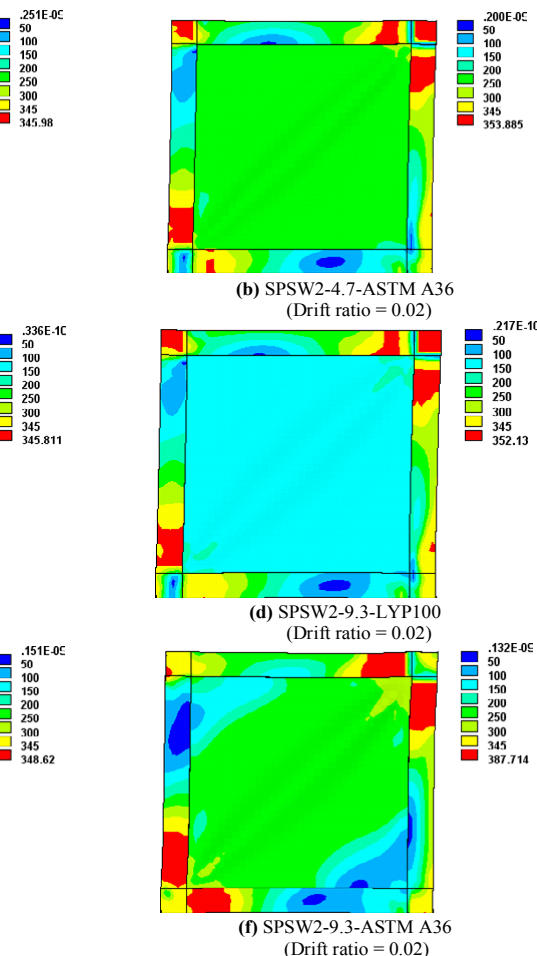

Figure 7: Von Mises stress contour plots of SPSW2 model with various infil plate thicknesses and steel types at drift ratios of 0.01 and 0.02 .

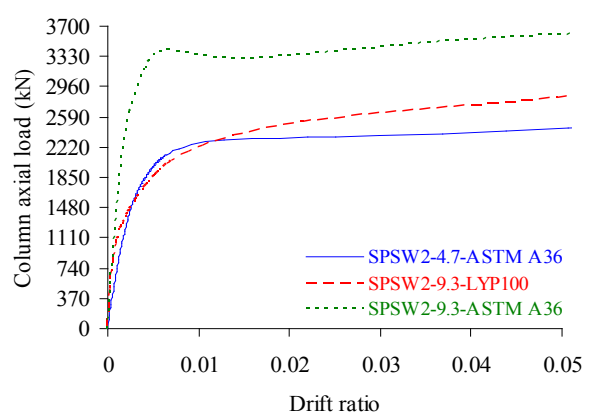

Figure 8: Comparison of the developed column axial loads.

from comparison of the stress contour plots between the SPSW2-9.3LYP100 (Figures 7c and 7d) and SPSW2-9.3-ASTM A36 Figure 7e and $7 \mathrm{f}$ models that the application of LYP steel infill plates results in a larger energy dissipation capacity over its conventional steel counterpart since yielding of the material in the former case is more extensively distributed over the entire plate component.

\section{Axial load in vertical boundary members}

Considering the axial loads developed in the columns of the SPSW2-4.7-ASTM A36 and SPSW2-9.3-LYP100 models, as shown in Figure 8, reveals a similarity in the overall performance of the two systems. Interestingly, applying LYP steel infill plates with twice the thickness does not increase the column axial load; doubling the thickness of conventional steel infill plates, however, does result in an increased column axial load capacity. 
Citation: Khoeilar AR, Zirakian T, Boyajian D, Maalouf S, Dermendjian N (2015) Case Study on Retrofit of Steel Plate Shear Walls Using Low Yield Point Steel Infill Plates. J Steel Struct Constr 2: 106. doi:10.4172/2472-0437.1000106

Page 5 of 6

\begin{tabular}{|c|c|c|c|c|c|c|c|c|c|}
\hline Cycle No. & $\mathbf{1}$ & $\mathbf{2}$ & $\mathbf{3}$ & $\mathbf{4}$ & $\mathbf{5}$ & $\mathbf{6}$ & $\mathbf{7}$ & $\mathbf{8}$ \\
\hline Drift ratio & 0.001 & 0.0025 & 0.005 & 0.01 & 0.015 & 0.02 & 0.03 \\
\hline
\end{tabular}

Table 2: Loading protocol.

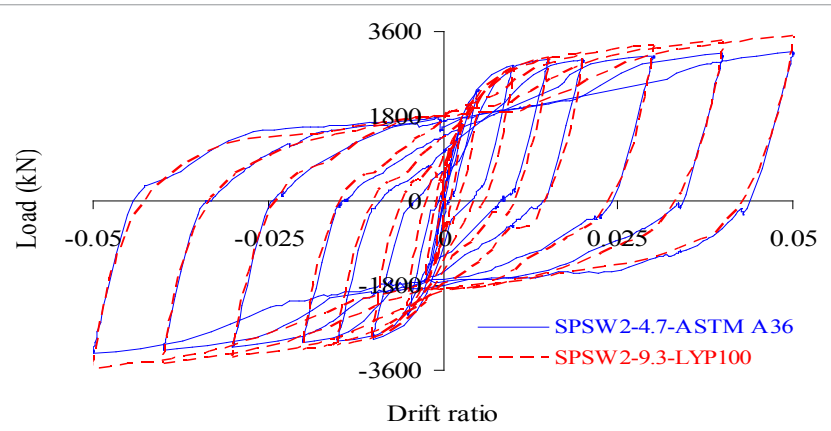

Figure 9: Comparison of the hysteretic behaviors.

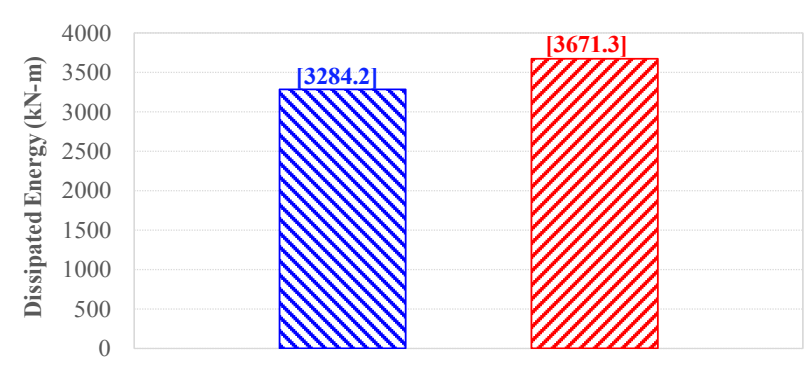

SPSW2-4.7-ASTMA36 SPSW2-9.3-LYP100

Figure 10: Total cumulative energies dissipated by SPSW2-4.7-ASTM A36 and SPSW2-9.3-LYP100 models.

\section{Hysteretic behavior}

The hysteretic behaviors of the SPSW2-4.7-ASTM A36 and SPSW2-9.3-LYP100 models with similar structural characteristics were evaluated by performing nonlinear cyclical analyses. The cyclic loading protocol used is furnished in Table 2 and Figure 9 displays hysteresis curves of both SPSW models.

From Figure 9 it is clear that both SPSW models have similar hysteretic behaviors. The total cumulative energies dissipated by the SPSW2-4.7-ASTM A36 and SPSW2-9.3-LYP100 models are 3284.2 and $3671.3 \mathrm{kN}-\mathrm{m}$ as shown in Figure 10, respectively, indicating that the LYP model possesses a greater energy absorption capacity $(\approx 12 \%)$. These findings indicate that the $4.7 \mathrm{~mm}$ ASTM A36 steel infill plate may be replaced by a double thickness $9.3 \mathrm{~mm}$ LYP100 steel plate with improved enhancements of initial stiffness, buckling characteristics, energy dissipation capacities, and serviceability. It is also worth noting that use of LYP steel infill plates with lower slenderness ratios does not increase the overall system demand on the boundary frame members while effectively assuaging stiffness and over-strength concerns associated with conventional steel plates.

\section{Conclusion}

The advantages of application of a LYP steel infill plate with double thickness in seismic retrofit of a conventional steel shear wall system were demonstrated in this paper through detailed numerical simulations.
It was found that the low yielding strength of a LYP steel infill plate with fairly large thickness favorably results in early yielding of the infill plate relative to the frame yielding. It was also shown that application of a LYP steel infill plate with double thickness can result in desirable strength performance and improved initial stiffness of the retrofitted SPSW system. The findings of this study, also, showed that the early yielding of the LYP steel infill plate with double thickness results in favorable plate-frame interaction and improves the hysteretic performance of the retrofitted SPSW system.

Overall, the application of the considered retrofit strategy, i.e., replacement of a conventional steel infill plate by a LYP steel plate with double thickness, is shown to be quite effective in improving the structural behavior and seismic performance of the existing steel shear wall systems. Furthermore, employment of infill plates made of LYP steel with exclusive material properties for seismic applications can facilitate the design and improve the buckling stability, serviceability, and energy absorption capacity of SPSW systems.

\section{References}

1. Sabouri-Ghomi S, Ventura CE, Kharrazi MHK (2005) Shear analysis and design of ductile steel plate walls. Journal of Structural Engineering 131: 878-889.

2. Esfandiar R, Barkhordari MA (2008) Investigation of behaviour of thin steel plate shear walls. Proceedings of the 14th World Conference on Earthquake Engineering, Beijing, China.

3. Chen SJ, Jhang C (2011) Experimental study of low-yield-point steel plate shear wall under in-plane load. Journal of Constructional Steel Research 67: 977-985.

4. Kulak GL, Kennedy DJL, Driver RG, Medhekar M (2001) Steel plate shear walls - An overview. Engineering Journal.

5. Bruneau M, Bhagwagar $\mathrm{T}$ (2002) Seismic retrofit of flexible steel frames using thin infill panels. Engineering Structures 24: 443-453.

6. De Matteis G, Landolfo R, Mazzolani FM (2003) Seismic response of MR steel frames with low-yield steel shear panels. Engineering Structures 25: 155-168.

7. Vian D, Bruneau M (2004) Testing of special LYS steel plate shear walls Proceedings of the $13^{\text {th }}$ World Conference on Earthquake Engineering Vancouver, B.C, Canada.

8. Tsai KC, Lin YC (2005) Seismic performance of steel plate shear wall frames. Proceedings of the 4th International Conference on Advances in Steel Structures, Shanghai, China.

9. Lashgari M (2009) Finite element analysis of thin steel plate shears walls World Academy of Science Engineering and Technology.

10. Mistakidis E (2010) Numerical study of low-yield point steel shear walls used for seismic applications. Engineering Computations: International Journal for Computer-Aided Engineering and Software 27: 257-279.

11. Chen SJ, Jhang C (2006) Cyclic behavior of low yield point steel shear walls Thin-Walled Structures 44: 730-738.

12. Zirakian T, Zhang J (2015) Buckling and yielding behavior of unstiffened slender moderate and stocky low yield point steel plates. Thin-Walled Structures 88: 105-118.

13. Zirakian T, Zhang J (2015) Seismic design and behavior of low yield point steel plate shear walls. International Journal of Steel Structures 15: 135-151.

14. Zhang J, Zirakian T (2015) Probabilistic assessment of structures with SPSW systems and LYP steel infill plates using fragility function method. Engineering Structures 85: 195-205.

15. Faella C, Lima C, Martinelli E, Realfonzo R (2014) Steel bracing configurations for seismic retrofitting of a reinforced concrete frame. Proceedings of the Institution of Civil Engineers - Structures and Buildings 167: 54-65. 
Citation: Khoeilar AR, Zirakian T, Boyajian D, Maalouf S, Dermendjian N (2015) Case Study on Retrofit of Steel Plate Shear Walls Using Low Yield Point Steel Infill Plates. J Steel Struct Constr 2: 106. doi:10.4172/2472-0437.1000106

Page 6 of 6

16. Gheitasi A, Alinia MM (2010) Slenderness classification of unstiffened metal plates under shear loading. Thin-Walled Structures 48: 508-518.

17. AISC 341-10 (2010) Seismic Provisions for Structural Steel Buildings. American Institute of Steel Construction, Chicago, IL.
18. Sabelli R, Bruneau M (2006) Steel Plate Shear Walls. Steel Design Guide 20, American Institute of Steel Construction, Chicago, IL.

19. ANSYS 11.0 (2007) ANSYS 11.0 documentation, ANSYS Inc.

20. Lubell AS (1997) Performance of Unstiffened Steel Plate Shear Walls Under Cyclic Quasi-Static Loading. Department of Civil Engineering, University of British Columbia, Canada. 